\title{
ON ADDITIVE SOLUTIONS OF A LINEAR EQUATION
}

\author{
A. VARGA ${ }^{1}$
}

ABSTRACT. In this paper we investigate the functional equation

$$
\sum_{i=1}^{n} \alpha_{i} A\left(\beta_{i} x\right)=0
$$

which holds for all $x \in \mathbb{R}$ with an unknown additive function $A: \mathbb{R} \rightarrow \mathbb{R}$ and fixed real parameters $\alpha_{i}, \beta_{i}$, where $i=1, \ldots, n$. Here we give sufficient and necessary conditions for the existence of non-trivial additive solutions of equation above in some cases depending on the algebraic properties of the parameters.

\section{INTRODUCTION AND PRELIMINARIES}

Consider the functional equation

$$
\sum_{i=1}^{n} \alpha_{i} A\left(\beta_{i} x\right)=0
$$

which holds for all $x \in \mathbb{R}$ (the reals) with an unknown additive function $A: \mathbb{R} \rightarrow \mathbb{R}$ and fixed real parameters $\alpha_{i}, \beta_{i}$, where $i=1, \ldots, n$. Since for any additive function vanishes at $x=0$, without loss of generality we can suppose that none of the parameters equals to zero.

The theory of functional equations containing weighted arithmetic means gives motivations to investigate (1.1) (see [4]).

(1.1) has been investigated for the case $n=2$ by Daróczy [2]. His fundamental result states that the functional equation

$$
\alpha_{1} A\left(\beta_{1} x\right)+\alpha_{2} A\left(\beta_{2} x\right)=0
$$

has a non-trivial additive solution $A$ if and only if both the parameters

$$
\alpha:=-\frac{\alpha_{2}}{\alpha_{1}} \text { and } \beta:=\frac{\beta_{1}}{\beta_{2}}
$$

2000 Mathematics Subject Classification. 39B22.

Key words and phrases. Functional equation, additive functions.

This research has been supported by the Hungarian Scientific Research Fund (OTKA) Grant NK-68040. 
are transcendent or they are algebraic with the same defining polynomial over $\mathbb{Q}$ (the rationals). Equation (1.2) is equivalent to

$$
A(\beta x)=\alpha A(x) \quad(x \in \mathbb{R})
$$

i.e. the solutions must be semi-homogeneous. This is a motivation to find conditions for the existence of non-trivial semi-homogeneous solutions of equation (1.1) for the case $n \geq 3$. It is easy to see that equation (1.1) is equivalent to the equation

$$
\sum_{i=1}^{n} \frac{\alpha_{i}}{\alpha_{n}} A\left(\frac{\beta_{i}}{\beta_{n}} x\right)=0 \quad(x \in \mathbb{R}) .
$$

In terms of the parameters $\frac{\alpha_{1}}{\alpha_{n}}, \ldots, \frac{\alpha_{n-1}}{\alpha_{n}}$ and $\frac{\beta_{1}}{\beta_{n}}, \ldots, \frac{\beta_{n-1}}{\beta_{n}}(n \geq 3)$ sufficient conditions can be found for the existence of non-trivial semihomogeneous additive solutions in [3]. In connection with these results we need the following notions.

Definition 1.1. Let $m$ be a positive integer and consider the element $\vec{\lambda}:=\left(\lambda_{1}, \ldots, \lambda_{m}\right)$ of the coordinate space $\mathbb{R}^{m}$. If the ideal

$$
\mathcal{I}(\vec{\lambda}):=\left\{p \in \mathbb{Q}\left[x_{1}, \ldots, x_{m}\right] \mid p\left(\lambda_{1}, \ldots, \lambda_{m}\right)=0\right\}
$$

of the polynomial ring $\mathbb{Q}\left[x_{1}, \ldots, x_{m}\right]$ contains only the zero polynomial we say that the coordinates $\lambda_{1}, \ldots, \lambda_{m}$ are algebraically independent. Otherwise they are algebraically dependent.

Theorem 1.2. Suppose that $n \geq 3$. If the parameters $\frac{\beta_{1}}{\beta_{n}}, \ldots, \frac{\beta_{n-1}}{\beta_{n}}$ are algebraically independent and at least one of the parameters $\frac{\alpha_{1}}{\alpha_{n}}, \ldots, \frac{\alpha_{n-1}}{\alpha_{n}}$ is transcendent then equation (1.1) has non-trivial additive solutions which are semi-homogeneous in the sense that

$$
A\left(\frac{\beta_{i}}{\beta_{n}} x\right)=\delta_{i} A(x)
$$

for some $\delta_{i}$ 's, for all $x \in \mathbb{R}$ and $i=1, \ldots n-1$.

Theorem 1.3. Suppose that $n \geq 3$. If the parameters $\frac{\alpha_{1}}{\alpha_{n}}, \ldots, \frac{\alpha_{n-1}}{\alpha_{n}}$ are algebraically independent and at least one of the parameters $\frac{\beta_{1}}{\beta_{n}}, \ldots, \frac{\beta_{n-1}}{\beta_{n}}$ is transcendent then equation (1.1) has non-trivial additive solution which is semi-homogeneous in the sense that

$$
A\left(\delta_{i} x\right)=\frac{\alpha_{i}}{\alpha_{n}} A(x)
$$

for some $\delta_{i}$ 's, for all $x \in \mathbb{R}$ and $i=1, \ldots n-1$. 
For example the coordinates of $(\sqrt{\pi}, 2 \pi+1)$ are algebraically dependent over $\mathbb{Q}$, since the non-zero polynomial $P\left(x_{1}, x_{2}\right)=2 x_{1}^{2}-x_{2}+1$ vanishes at $(\sqrt{\pi}, 2 \pi+1)$.

The Lindemann-Weierstrass theorem gives a method to construct algebraically independent systems (see [1], Theorem 1.4. p.6.). It says that if $\lambda_{1}, \ldots, \lambda_{n}$ are algebraic numbers such that they are linearly independent over $\mathbb{Q}$, then

$$
e^{\lambda_{1}}, \ldots, e^{\lambda_{n}}
$$

are algebraically independent over $\mathbb{Q}$.

In this paper we give sufficient and necessary conditions for the existence of non-trivial additive solutions of (1.1) under some conditions like in Theorem 1.1 and Theorem 1.2.

\section{Gauss elimination pRocess FOR a System of EQUATIONS CONTAINING AN UNKNOWN ADDITIVE FUNCTION}

The main tool of our investigations is the following

Theorem 2.1. Let $k$ be a natural number such that $k \geq 2$. Furthermore, let $u, a_{i j}$ be fixed real numbers $(i, j=1, \ldots, k)$. If the matrix $M_{1}:=\left(a_{i j}\right)_{k \times k}$ is regular, then the only additive function $A: \mathbb{R} \rightarrow \mathbb{R}$ that satisfies the system of equations

$$
u^{k-1} A\left(a_{i 1} x\right)+u^{k-2} A\left(a_{i 2} x\right)+\ldots+u A\left(a_{i k-1} x\right)+A\left(a_{i k} x\right)=0
$$

$(x \in \mathbb{R} ; i=1, \ldots, k)$ is the identically zero function.

Proof. For the simplicity $M_{1}$ will be called the matrix of the system (2.1), and the equation

$$
u^{k-1} A\left(\mu a_{i 1} x\right)+u^{k-2} A\left(\mu a_{i 2} x\right)+\ldots+u A\left(\mu a_{i k-1} x\right)+A\left(\mu a_{i k} x\right)=0
$$

will be denoted by $E_{i(\mu x)}^{k-1}$ for any indeces $i=1, \ldots, k$ and $\mu \in \mathbb{R}$.

In the proof we imitate the steps of the Gauss elimination process. Without loss of generality we may assume that $a_{11} \neq 0$ because the rank of $M_{1}$ is maximal. Taking the difference $E_{i\left(a_{11} x\right)}^{k-1}-E_{1\left(a_{i 1} x\right)}^{k-1}(i=2, \ldots, k)$ and using the additivity of $\mathrm{A}$ we get that

$$
\begin{gathered}
u^{k-2} A\left(\left(a_{11} a_{i 2}-a_{12} a_{i 1}\right) x\right)+\ldots+u A\left(\left(a_{11} a_{i k-1}-a_{1 k-1} a_{i 1}\right) x\right)+ \\
+A\left(\left(a_{11} a_{i k}-a_{1 k} a_{i 1}\right) x\right)=0
\end{gathered}
$$

holds for all $x \in \mathbb{R}$ and for all indeces $i=2, \ldots, k$. Equations (2.2) and $E_{1(x)}^{k-1}$ form a system of equations which has the matrix 


$$
M_{2}=\left(\begin{array}{ccccc}
a_{11} & a_{12} & \cdot & \cdot & a_{1 k} \\
0 & a_{11} a_{22}-a_{12} a_{21} & \cdot & \cdot & \cdot \\
\cdot & \cdot & \cdot & \cdot & \cdot \\
\cdot & \cdot & \cdot & \cdot & \cdot \\
0 & a_{11} a_{k 2}-a_{12} a_{k 1} & \cdot & \cdot & a_{11} a_{k k}-a_{1 k} a_{k 1}
\end{array}\right)
$$

We may suppose that $a_{11} a_{22}-a_{12} a_{21} \neq 0$ because of the regularity of $M_{1}$. Continuing this process, in the $(k-1)^{s t}$ step the matrix $M_{1}$ becomes of triangular form $M_{k}:=\left(\lambda_{i j}\right)_{k \times k}$ for some $\lambda_{i j} \in \mathbb{R}$ with $\lambda_{i j}=0$ if $i>j$. Since $0 \neq \operatorname{det} M_{1}=\operatorname{det} M_{k}=\prod_{i=1}^{k} \lambda_{i i}$ and the last equation of the system obtained in the $(k-1)^{s t}$ step is $A\left(\lambda_{k k} x\right)=0$ for all $x \in \mathbb{R}$ we get that $A$ is the identically zero function.

\section{The MAIN RESUlT}

The new part of the main result of this paper is the following

Theorem 3.1. Suppose that $n \geq 3$. If the parameters $\frac{\beta_{1}}{\beta_{n}}, \ldots, \frac{\beta_{n-1}}{\beta_{n}}$ are algebraically independent and all of the parameters $\frac{\alpha_{1}}{\alpha_{n}}, \ldots, \frac{\alpha_{n-1}}{\alpha_{n}}$ are algebraic then the only additive solution of equation (1.1) is the identically zero function.

Proof. For the simplicity we use the notations

$$
\omega_{i}:=\frac{\alpha_{i}}{\alpha_{n}}, \quad \xi_{i}:=\frac{\beta_{i}}{\beta_{n}} \quad(i=1, \ldots, n-1) .
$$

As $\omega_{i}(i=1, \ldots, n-1)$ is algebraic over $\mathbb{Q}$, therefore $\mathbb{Q}\left(\omega_{1}, \ldots, \omega_{n-1}\right)$ is a finite extension of $\mathbb{Q}$. Moreover $\mathbb{Q}$ is a field with zero characteristic, thus there exist $u \in \mathbb{R}$ such that $\mathbb{Q}(u)=\mathbb{Q}\left(\omega_{1}, \ldots, \omega_{n-1}\right)$. Let $k$ be the degree of extension. As $1, u, \ldots, u^{k-1}$ is a basis of the vector space $\mathbb{Q}(u)$, thus $\omega_{i}=\sum_{j=0}^{k-1} r_{i j} u^{j}$ with some $r_{i j} \in \mathbb{Q}(i=1, \ldots, n-1 ; j=$ $0, \ldots, k-1)$. Using this form of $\omega_{i}$, equation (1.1) goes over into the equation

$$
u^{k-1} A\left(p_{k-1} x\right)+u^{k-2} A\left(p_{k-2} x\right)+\ldots+u A\left(p_{1} x\right)+A\left(p_{0} x\right)=0
$$

where $p_{j}=\sum_{i=1}^{n-1} \xi_{i} r_{i j}(j=1, \ldots, k-1)$ and $p_{0}=1+\sum_{i=1}^{n-1} \xi_{i} r_{i 0}$. It is easy to see that $p_{0} \neq 0$. (Otherwise $\xi_{1}, \ldots, \xi_{n-1}$ can not be algebraically independent.) Let

$$
f(t):=t^{k}+q_{k-1} t^{k-1}+\ldots+q_{1} t+q_{0} \quad\left(q_{i} \in \mathbb{Q}, \quad i=0, \ldots, k-1\right)
$$

be the defining polynomial of $u$. Then we have that

$$
u^{k}=-\left(q_{k-1} u^{k-1}+\ldots+q_{1} u+q_{0}\right) .
$$


Multiplying both sides of (3.1) by $\mathrm{u}$ and using (3.2), the additivity and rational homogeneity of $A$ we get that

$$
\begin{gathered}
u^{k-1} A\left(\left(p_{k-2}-q_{k-1} p_{k-1}\right) x\right)+\ldots+u^{2} A\left(\left(p_{1}-q_{2} p_{k-1}\right) x\right)+ \\
+u A\left(\left(p_{0}-q_{1} p_{k-1}\right) x\right)+A\left(-q_{0} p_{k-1} x\right)=0 .
\end{gathered}
$$

Multiplying both sides of (3.3) by $\mathrm{u}$ and using the process above $k-1$ times we get a system of equations of type (2.1). So, in what follows, it is enough to prove that the matrix $M:=\left(b_{i j}\right)_{k \times k}$ of this system is regular, since by Theorem (2.1), $A=0$.

Suppose, in contrary, that $\operatorname{det} M=0$. One can easily check that $b_{i j}=z^{(i j)}\left(\xi_{1}, \ldots, \xi_{n-1}\right)$ for some $z^{(i j)} \in \mathbb{Q}\left[x_{1}, \ldots, x_{n-1}\right](i, j=1, \ldots, k)$ therefore

$$
\operatorname{det} M=z\left(\xi_{1}, \ldots, \xi_{n-1}\right)
$$

where $z \in \mathbb{Q}\left[x_{1}, \ldots, x_{n-1}\right]$ is defined by

$$
\begin{gathered}
z\left(x_{1}, \ldots, x_{n-1}\right)= \\
=\operatorname{det}\left(\begin{array}{cccc}
z^{(11)}\left(x_{1}, \ldots, x_{n-1}\right) & \cdot & \cdot & \\
\cdot & & z^{(i k-i+1)}\left(x_{1}, \ldots, x_{n-1}\right) & \cdot \\
\cdot & \cdot & \cdot & \cdot \\
\cdot & . & \cdot & z^{(k k)}\left(x_{1}, \ldots, x_{n-1}\right)
\end{array}\right) .
\end{gathered}
$$

We show that $z$ is not the identically zero polynomial which contradicts to the algebraic independence of $\xi_{1}, \ldots, \xi_{n-1}$.

To see this we show that $z^{(i j)}$ does not vanish at the zero vector if $j=k-i+1(i=1, \ldots, k)$, moreover in this case $z^{(i j)}(0, \ldots, 0)=1$, otherwise it vanishes. The proof of this statement goes by induction on $i$. If $i=1$ then the statement is true, because

$$
\begin{gathered}
z^{(1 j)}\left(x_{1}, \ldots, x_{n-1}\right)=\sum_{i=1}^{n-1} r_{i j} x_{i} \text { thus } z^{(1 j)}(0, \ldots, 0)=0(j=1, \ldots, k-1), \\
z^{(1 k)}\left(x_{1}, \ldots, x_{n-1}\right)=1+\sum_{i=1}^{n-1} r_{i 0} x_{i} \text { thus } z^{(1 k)}(0, \ldots, 0)=1 .
\end{gathered}
$$

Assume that, for $2 \leq i \leq k-1$,

$z^{(1 j)}(0, \ldots, 0)=0$ if $j \neq k-i+1 \quad(j=1, \ldots, k)$ and $z^{(i k-i+1)}(0, \ldots, 0)=1$

hold. Now we look for connection between the coefficients $b_{i+1} j$ and $b_{i l}(j, l=1, \ldots, k)$.

Since the $i^{\text {th }}$ equation of the system is

$$
u^{k-1} A\left(b_{i 1} x\right)+\ldots+u^{i-1} A\left(b_{i k-i+1} x\right)+\ldots+A\left(b_{i k} x\right)=0
$$


while the $(i+1)^{s t}$ one is

$$
-\left(q_{k-1} u^{k-1}+\ldots+q_{i} u^{i}+\ldots+q_{0}\right) A\left(b_{i 1} x\right)+\ldots+u^{i} A\left(b_{i k-i+1} x\right)+\ldots+u A\left(b_{i k} x\right)=0,
$$

that is,

$u^{k-1} A\left(\left(b_{i 2}-q_{k-1} b_{i 1}\right) x\right)+\ldots+u^{i} A\left(\left(b_{i k-i+1}-q_{i} b_{i 1}\right) x\right)+\ldots+A\left(q_{0} b_{i 1} x\right)=0$,

we get that $b_{i+1}{ }_{j}=b_{i j+1}-q_{k-j} b_{i 1}(j=1, \ldots, k)$. So, we have that $z^{(i+1 j)}=z^{(i j+1)}-q_{k-j} z^{(i 1)}(j=1, \ldots, k)$.

If $j=k-i$ then $z^{(i+1 k-i)}=z^{(i k-i+1)}-q_{i} z^{(i 1)}$. Since $z^{(i 1)}(0, \ldots, 0)=0$ and $z^{(i k-i+1)}(0, \ldots, 0)=1$ it follows that $z^{(i+1 k-i)}(0, \ldots, 0)=1$.

If $j \neq k-i$ then $z^{(i j+1)}(0, \ldots, 0)=0$ thus $z^{(i+1 j)}(0, \ldots, 0)=0$.

According to the facts above it follows that

$$
z(0, \ldots, 0)=\operatorname{det}\left(\begin{array}{cccccc}
0 & \cdot & \cdot & \cdot & 0 & 1 \\
\cdot & & & 1 & 0 \\
\cdot & & & \cdot & & \cdot \\
\cdot & & . & & \cdot \\
0 & 1 & & & & \cdot \\
1 & 0 & . & . & . & 0
\end{array}\right)=(-1)^{\frac{k(k+3)}{2}}
$$

which implies that $z$ is not the identically zero polynomial.

The following theorem says that the role of the inner and outher parameters can be interchanged in Theorem 3.1.

Theorem 3.2. Suppose that $n \geq 3$. If the parameters $\frac{\alpha_{1}}{\alpha_{n}}, \ldots, \frac{\alpha_{n-1}}{\alpha_{n}}$ are algebraically independent and all of the parameters $\frac{\beta_{1}}{\beta_{n}}, \ldots, \frac{\beta_{n-1}}{\beta_{n}}$ are algebraic then the only additive solution of equation (1.1) is the identically zero function.

Proof. The proof is similar to that of the previous theorem. With the notations $\omega_{i}:=\frac{\alpha_{i}}{\alpha_{n}}, \quad \xi_{i}:=\frac{\beta_{i}}{\beta_{n}}(i=1, \ldots, n-1)$ we get that there exists $u \in \mathbb{R}$ such that $\mathbb{Q}(u)=\mathbb{Q}\left(\xi_{1}, \ldots, \xi_{n-1}\right)$. If the degree of the extension is equal to $k$ then $1, u, \ldots, u^{k-1}$ is a basis of $\mathbb{Q}(u)$, thus $\xi_{i}=\sum_{j=0}^{k-1} r_{i j} u^{j}$ with some $r_{i j} \in \mathbb{Q}(i=1, \ldots, n-1 ; j=0, \ldots, k-1)$. Using this form of $\xi_{i}$ equation (1.1) goes over into the equation

$$
p_{k-1} A\left(u^{k-1} x\right)+p_{k-2} A\left(u^{k-2} x\right)+\ldots+p_{1} A(u x)+p_{0} A(x)=0
$$

where $p_{j}=\sum_{i=1}^{n-1} \omega_{i} r_{i j}(j=1, \ldots, k-1)$ and $p_{0}=1+\sum_{i=1}^{n-1} \omega_{i} r_{i 0}$. It is easy to see, that $p_{0} \neq 0$. Using again the notation

$$
f(t):=t^{k}+q_{k-1} t^{k-1}+\ldots+q_{1} t+q_{0} \quad\left(q_{i} \in \mathbb{Q}, \quad i=0, \ldots, k-1\right)
$$

for the defining polynomial of $u$ we can use again the equality (3.2). Replace $x$ by $u x$ in (3.4). Using (3.2), the additivity and rational 
homogeneity of $A$ we get that

$$
\begin{gathered}
\left(p_{k-2}-q_{k-1} p_{k-1}\right) A\left(u^{k-1} x\right)+\ldots+\left(p_{1}-q_{2} p_{k-1}\right) A\left(u^{2} x\right)+ \\
+\left(p_{0}-q_{1} p_{k-1}\right) A(u x)+\left(-q_{0} p_{k-1}\right) A(x)=0 .
\end{gathered}
$$

Replace $x$ by $u^{2} x, \ldots, u^{k-1} x$, respectively in equation (3.4). Then we get a system of equations with $k$ unknowns and $k$ equations which has the matrix form

$$
L\left(\begin{array}{c}
A\left(u^{k-1} x\right) \\
\cdot \\
\cdot \\
A(u x) \\
A(x)
\end{array}\right)=0
$$

where $L:=\left(c_{i j}\right)_{k \times k}$ consists of the coefficients of the system. We are going to prove that $\operatorname{det} L \neq 0$ which means that the linear transformation represented by $L$ is regular and the kernel contains only the zero vector. Hence $A(x)=0$ for any $x \in \mathbb{R}$.

Suppose, in contrary that $\operatorname{det} L=0$. It is easy to see that

$c_{i j}=z^{(i j)}\left(\omega_{1}, \ldots, \omega_{n-1}\right)$ for some $z^{(i j)} \in \mathbb{Q}\left[x_{1}, \ldots, x_{n-1}\right] \quad(i, j=1, \ldots, k)$.

Therefore $\operatorname{det} L=z\left(\omega_{1}, \ldots, \omega_{n-1}\right)$ where $z \in \mathbb{Q}\left[x_{1}, \ldots, x_{n-1}\right]$ is defined as in the proof of Theorem 3.1. Like in the proof of Theorem 3.1, we can show that

$$
z^{(i j)}(0, \ldots, 0)=0 \text { if } j \neq k-i+1 \quad(i, j=1, \ldots, k) \text { and } z^{(i k-i+1)}(0, \ldots, 0)=1,
$$

which implies that $z(0, \ldots, 0)=(-1)^{\frac{k(k+3)}{2}}$. This means that $z$ is not the identically zero polynomial which contradicts to the algebraic independence of $\omega_{1}, \ldots, \omega_{n-1}$. (3.6) can be proved by induction on $i$ using the connection of coefficients $c_{i+1} j$ and $c_{i l}$. As it was in the proof of Theorem 3.1 this connection can easily be found if we consider the $i^{\text {th }}$ equation

$$
c_{i 1} A\left(u^{k-1} x\right)+\ldots+c_{i k-i+1} A\left(u^{i-1} x\right)+\ldots+c_{i k} A(x)=0
$$

and the $(i+1)^{s t}$ one

$\left(c_{i 2}-q_{k-1} c_{i 1}\right) A\left(u^{k-1} x\right)+\ldots+\left(c_{i k-i+1}-q_{i} c_{i 1}\right) A\left(u^{i} x\right)+\ldots+q_{0} c_{i 1} A(x)=0$

from the system of equations.

Combining Theorems 1.1 and 3.1, Theorems 1.2 and 3.2, respectively we have the following main results.

Theorem 3.3. Suppose that $n \geq 3$ and the parameters $\frac{\beta_{1}}{\beta_{n}}, \ldots, \frac{\beta_{n-1}}{\beta_{n}}$ are algebraically independent. There exist a not identically zero additive solution of equation (1.1) if and only if at least one of the parameters $\frac{\alpha_{1}}{\alpha_{n}}, \ldots, \frac{\alpha_{n-1}}{\alpha_{n}}$ is transcendent. 
Theorem 3.4. Suppose that $n \geq 3$ and the parameters $\frac{\alpha_{1}}{\alpha_{n}}, \ldots, \frac{\alpha_{n-1}}{\alpha_{n}}$ are algebraically independent. There exist a not identically zero additive solution of equation (1.1) if and only if at least one of the parameters $\frac{\beta_{1}}{\beta_{n}}, \ldots, \frac{\beta_{n-1}}{\beta_{n}}$ is transcendent.

\section{About the Remaining Case}

To complete the discussion we need to investigate the case when both

of the collections of the parameters $\frac{\beta_{1}}{\beta_{n}}, \ldots, \frac{\beta_{n-1}}{\beta_{n}}$ and $\frac{\alpha_{1}}{\alpha_{n}}, \ldots, \frac{\alpha_{n-1}}{\alpha_{n}}$ are algebraically dependent. In case of a special functional equation of the form (1.1) we give necessary and sufficient conditions for the existence of non-trivial solutions.

Theorem 4.1. Let $n=3, \beta_{1}=\sqrt{d_{1}}, \beta_{2}=\sqrt{d_{2}}, \beta_{3}=1$ in (1.1) where $d_{1}$ and $d_{2}$ are positive rational numbers such that $\sqrt{d_{1}}$ and $\sqrt{d_{2}}$ are irrationals. Furthermore, suppose that $\alpha_{1} \alpha_{2} \alpha_{3} \neq 0$. The functional equation

$$
\alpha_{1} A\left(\sqrt{d_{1}} x\right)+\alpha_{2} A\left(\sqrt{d_{2}} x\right)+\alpha_{3} A(x)=0
$$

has non-trivial additive solutions if and only if one of the conditions

$$
\begin{aligned}
& \text { (i) } 1+\frac{\alpha_{1}}{\alpha_{3}} \sqrt{d_{1}}+\frac{\alpha_{2}}{\alpha_{3}} \sqrt{d_{2}}=0, \\
& \text { (ii) } 1+\frac{\alpha_{1}}{\alpha_{3}} \sqrt{d_{1}}-\frac{\alpha_{2}}{\alpha_{3}} \sqrt{d_{2}}=0, \\
& \text { (iii) } 1-\frac{\alpha_{1}}{\alpha_{3}} \sqrt{d_{1}}+\frac{\alpha_{2}}{\alpha_{3}} \sqrt{d_{2}}=0, \\
& \text { (iv) } 1-\frac{\alpha_{1}}{\alpha_{3}} \sqrt{d_{1}}-\frac{\alpha_{2}}{\alpha_{3}} \sqrt{d_{2}}=0 .
\end{aligned}
$$

is satisfied.

Proof. Suppose that $A$ is not the identically zero function. Equation (4.1) is equivalent to the equation

$$
\frac{\alpha_{1}}{\alpha_{3}} A\left(\sqrt{d_{1}} x\right)+\frac{\alpha_{2}}{\alpha_{3}} A\left(\sqrt{d_{2}} x\right)+A(x)=0 .
$$

Substituting $\sqrt{d_{1}} x, \sqrt{d_{2}} x$ and $\sqrt{d_{1} d_{2}} x$ in (4.2), respectively we get the system of equations which has the matrix form

$$
M\left(\begin{array}{c}
A(x) \\
A\left(\sqrt{d_{1}} x\right) \\
A\left(\sqrt{d_{2}} x\right) \\
A\left(\sqrt{d_{1} d_{2}} x\right)
\end{array}\right)=\left(\begin{array}{l}
0 \\
0 \\
0 \\
0
\end{array}\right)
$$


where

$$
M:=\left(\begin{array}{cccc}
1 & \frac{\alpha_{1}}{\alpha_{3}} & \frac{\alpha_{2}}{\alpha_{3}} & 0 \\
d_{1} \frac{\alpha_{1}}{\alpha_{3}} & 1 & 0 & \frac{\alpha_{2}}{\alpha_{3}} \\
d_{2} \frac{\alpha_{2}}{\alpha_{3}} & 0 & 1 & \frac{\alpha_{1}}{\alpha_{3}} \\
0 & d_{2} \frac{\alpha_{2}}{\alpha_{3}} & d_{1} \frac{\alpha_{1}}{\alpha_{3}} & 1
\end{array}\right)
$$

Since

$$
\operatorname{det}\left(\begin{array}{ccc}
\frac{\alpha_{1}}{\alpha_{3}} & \frac{\alpha_{2}}{\alpha_{3}} & 0 \\
1 & 0 & \frac{\alpha_{2}}{\alpha_{3}} \\
0 & 1 & \frac{\alpha_{1}}{\alpha_{3}}
\end{array}\right)=-2 \frac{\alpha_{1} \alpha_{2}}{\alpha_{3}^{2}} \neq 0
$$

we have that the rank of $M$ is at least 3 . As $A$ is not the identically zero function the rank of $M$ cannot be maximal. So we may suppose that the rank of $M$ is 3 which implies that the kernel of the transformation represented by $M$ is one-dimensional, i.e. it is generated by a vector $\vec{v}=\left(v_{0}, v_{1}, v_{2}, v_{3}\right)$. This means that

$$
\left(\begin{array}{c}
A(x) \\
A\left(\sqrt{d_{1}} x\right) \\
A\left(\sqrt{d_{2}} x\right) \\
A\left(\sqrt{d_{1} d_{2}} x\right)
\end{array}\right)=\lambda(x)\left(\begin{array}{c}
v_{0} \\
v_{1} \\
v_{2} \\
v_{3}
\end{array}\right)
$$

for some additive function $\lambda: \mathbb{R} \rightarrow \mathbb{R}$ and $v_{0} \neq 0$. Thus we get that

$$
A\left(\sqrt{d_{1}} x\right)=v_{1} \lambda(x)=\frac{v_{1}}{v_{0}} A(x)
$$

and, in a similar way

$$
A\left(\sqrt{d_{2}} x\right)=v_{2} \lambda(x)=\frac{v_{2}}{v_{0}} A(x) .
$$

Both (4.3) and (4.4) has the form equation (1.2), therefore

$$
\frac{v_{1}}{v_{0}}= \pm \sqrt{d_{1}} \text { and } \frac{v_{2}}{v_{0}}= \pm \sqrt{d_{2}} \text {. }
$$

Thus one of the conditions (i), (ii), (iii) and (iv) is satisfied.

Conversely, if (i) is satisfied then, for example, let $A(x):=x$ or $A(x):=-x$. As an illustration consider the case when (ii) is satisfied. Now we prove that

$$
\vec{\mu}=\left(\sqrt{d_{1}}, \sqrt{d_{2}}\right) \text { and } \vec{\nu}=\left(\sqrt{d_{1}},-\sqrt{d_{2}}\right)
$$

have the same defining ideal, i.e. the ideals

$$
\mathcal{I}(\vec{\mu}):=\left\{P \in \mathbb{Q}\left[x_{1}, x_{2}\right] \mid P\left(\sqrt{d_{1}}, \sqrt{d_{2}}\right)=0\right\}
$$

and

$$
\mathcal{I}(\vec{\nu}):=\left\{P \in \mathbb{Q}\left[x_{1}, x_{2}\right] \mid P\left(\sqrt{d_{1}},-\sqrt{d_{2}}\right)=0\right\}
$$


are the same. Suppose that $P\left(\sqrt{d_{1}}, \sqrt{d_{2}}\right)=0$. Then we can write $P\left(\sqrt{d_{1}}, x_{2}\right)=f\left(x_{2}\right)\left(x_{2}^{2}-d_{2}\right)$ because the degree of $\sqrt{d_{2}}$ is 2 . Therefore $P\left(\sqrt{d_{1}},-\sqrt{d_{2}}\right)=0$. The method is similar for any elements $Q\left[x_{1}, x_{2}\right]$ of the defining ideal of $\vec{\nu}$. Therefore there exists a field isomorphism $\delta: \mathbb{Q}\left(\sqrt{d_{1}}, \sqrt{d_{2}}\right) \rightarrow \mathbb{Q}\left(\sqrt{d_{1}},-\sqrt{d_{2}}\right)$ such that $\delta\left(\sqrt{d_{1}}\right)=\sqrt{d_{1}}$ and $\delta\left(\sqrt{d_{2}}\right)=-\sqrt{d_{2}}$. For the proof see Lemma 3.1. in [4]. Now we can use the procedure of semilinear extension to construct a not identically zero additive function $A$ such that $A\left(\sqrt{d_{1}} x\right)=\sqrt{d_{1}} A(x)$ and $A\left(\sqrt{d_{2}} x\right)=-\sqrt{d_{2}} A(x)$. (See Theorem 3.2. in [4].) According to (ii) the function $A$ is obviously satisfies (4.1). The case of (iii) and (iv) is similar.

Example 4.2. The coordinates of $\left(e, \frac{\sqrt{d_{1}}}{\sqrt{d_{2}}} e+\frac{1}{\sqrt{d_{2}}}\right)$ are algebraically dependent over $\mathbb{Q}$, since the non-zero polynomial

$$
P\left(x_{1}, x_{2}\right)=\left(d_{1} x_{1}^{2}-d_{2} x_{2}^{2}-1\right)^{2}-4 d_{2} x_{2}^{2}
$$

vanishes at $\left(e, \frac{\sqrt{d_{1}}}{\sqrt{d_{2}}} e+\frac{1}{\sqrt{d_{2}}}\right)$. Theorem 4.1. implies that if $\frac{\alpha_{1}}{\alpha_{3}}=e$ and $\frac{\alpha_{2}}{\alpha_{3}}=\frac{\sqrt{d_{1}}}{\sqrt{d_{2}}} e+\frac{1}{\sqrt{d_{2}}}$, there exist non-trivial additive solutions of equation (4.1).

The coordinates of $\left(e, \frac{\sqrt{d_{1}}}{\sqrt{d_{2}}} e\right)$ are algebraically dependent over $\mathbb{Q}$, since the non-zero polynomial $P\left(x_{1}, x_{2}\right)=\frac{d_{1}}{d_{2}} x_{1}^{2}-x_{2}^{2}$ vanishes at $\left(e, \frac{\sqrt{d_{1}}}{\sqrt{d_{2}}} e\right)$. Theorem 4.1. implies that if $\frac{\alpha_{1}}{\alpha_{3}}=e$ and $\frac{\alpha_{2}}{\alpha_{3}}=\frac{\sqrt{d_{1}}}{\sqrt{d_{2}}} e$ then the only additive solution of (4.1) is the identically zero function.

\section{REFERENCES}

1. A. Baker, Transcendental number theory, Cambridge university Press 1975.

2. Z. Daróczy, Notwendige und hinreichende Bedingungen für die Existenz von nichtkonstanten Lösungen linearer Funktionalgleichungen, Acta Sci. Math. Szeged, 22 (1961), 31-41.

3. A. Varga, Cs. Vincze, On Daróczy's problem for additive functions, submitted to Publ. Math. Debrecen

4. A. Varga, Cs. Vincze On a functional equations containing weighted arithmetic means, ISNM Vol.157, 2009, 305-315

1 Institute of Mathematics, University of Debrecen, P. O. Box 12 , DEBRECEN, Hungary.

E-mail address: vargaa@math.klte.hu 